\title{
Intracellular protease activation in apoptosis and cell- mediated cytotoxicity characterized by cell-permeable fluorogenic protease substrates
}

\author{
Beverly Z Packard ${ }^{1}$, Akira Komoriya ${ }^{1}$ \\ ${ }^{1}$ OncoImmunin Inc., 207A Perry Parkway, Suite 6, Gaithersburg, MD 20877, USA
}

Over the past decade the importance of signaling from reporter molecules inside live cells and tissues has been clearly established. Biochemical events related to inflammation, tumor metastasis and proliferation, and viral infectivity and replication are examples of processes being further defined as more molecular tools for live cell measurements become available. Moreover, in addition to quantitating parameters related to physiologic processes, real-time imaging of molecular interactions that compose basic cellular activities are providing insights into understanding disease mechanisms as well as extending clinical efficacy of therapeutic regimens. In this review the use of highly cell-permeable fluorogenic substrates that report protease activities inside live cells is described; applications to defining the molecular events of two cellular processes, i.e., apoptosis and cell-mediated cytotoxicity, are then illustrated.

Keywords: protease, substrate, fluorescence, caspase, granzyme, apoptosis, cytotoxicity

Cell Research (2008) 18:238-247. doi: 10.1038/cr.2008.17; published online 29 January 2008

\section{Introduction}

Direct measurement of biologically active molecules in physiologically relevant environments is essential and has become of critical importance in the post genomics/ proteomics era. In contrast to vast databases of complete primary structures derivable from gene sequencing, data on molecular activities and functions are still relatively weak; furthermore, although the development of new bioinformatic tools has greatly improved the processing of information gathered from genomic and proteomic screening efforts, attempts to deduce structure/activity relationships by various sequence homology analyses of given genes within known families of proteins/genes can only yield potential properties of given gene products. Confirmation by direct activity measurements is essential. An area in which understanding the linkage between structure and function is particularly important is protease substrate and

Correspondence: Beverly Z Packard

Tel: +1-301-987-7881; Fax: +1-301-987-7882

E-mail: BPackard@PhiPhiLux.com

Abbreviations: antibody-dependent cellular cytotoxicity (ADCC); $\mathrm{CD} 8^{+}$ cytotoxic T lymphocyte (CTL); differential interference contrast (DIC); Granzyme B (GzB); monoclonal antibody (MAb); natural killer cell (NK cell); propidium iodide (PI); protein transduction domain (PTD) inhibitor design, as enzymes with 3-dimensional recognition and specific cleavage capability are central to virtually all physiologic processes.

In order to probe the topic of protease structure/function, three major issues must be confronted: (i) the linear versus 3-dimensional structure of proteases and protease targets, (ii) construction/modeling/reconstitution of physiologic environments in which proteolysis occurs and (iii) efficient means to transport reporter molecules into cells without disturbing the physiologic integrity of the system under study. More specific concerns regarding each of these issues are:

First, the information content of a predicted or actual linear amino acid sequence may be severely lacking when contrasted with the same sequence in its native environment as the latter provides a three-dimensional structure that the former lacks. Significantly, the three-dimensional constraints imposed on a sequence in a biochemically active macromolecule may be crucial for the accurate assessment of an enzyme, its physiologic substrates as well as inhibitors and, ultimately, design of new drugs.

Second, ex vivo analyses cannot identify physiologic neighbors or macromolecular targets, both essential for unraveling mechanisms at the whole cell level; nor can the impact of an intracellular milieu be assessed [1,2]. For example enzymes, which can exist in many, i.e., ac- 
tive, inactive, and latent, forms are often found in nature in complex with naturally occurring inhibitors; only upon an activating event such as a $\mathrm{pH}$ change or degranulation of a cytoplasmic compartment will an enzyme-inhibitor complex dissociate thereby enabling the enzyme to assume its active form.

The third represents an issue prevalent throughout science: how to measure a function without perturbing the function being measured. In the case of measuring protease activities in living systems efficient means for transport of reporter molecules into cells without disturbing the physiologic integrity of the system under study is the immediate issue. Therefore, intrinsic to the design of the probe must be a mechanism for entry and access to all environments of the cell. In order to achieve this goal the biophysical properties of many intracellular environments (cytosol, cytoplasmic organelles, and nucleus), the plasma membrane (three very distinct environments: the intracellular face, the transmembrane space, and the extracellular side), and the extracellular matrix must be taken into account.

Here we first present the background for protease activity probes, then proceed with a discussion addressing each of these issues with applications designed to test the proposed solutions.

\section{Approaches to protease probe and inhibitor design}

Protease specificity has been the subject of many studies and the methodology for optimizing sequences has advanced with the pace of instrument and software development. Existing techniques include identifying cleavage fragments of standard peptides or proteins following exposure to proteases of interest, creating combinatorial and context libraries, engineering phage displays, as well as genetic selection techniques using recently acquired bioinformatic knowledge [3]. However, despite the voluminous analytic database available, most protease activity probes suffer from the three issues described above: specificity, environmental context, and physiologic access.

These deficiencies derive from: (i) use of limited sequence information, often from only one side of a protease target's recognition/cleavage site and (ii) low permeability across membranes of live cells. For example, one class of protease probes uses a few, typically one to four, amino acids from the amino side of a target's recognition/cleavage site flanked by a group in the $\mathrm{P}_{1}{ }^{\prime}$ site that covalently binds to a protease's active site and in the $\mathrm{P}_{2,3,4, \text { or } 5}$ site a fluorophore or other amino terminal blocking group (Figure 1) [4]. Since probes with this design lack half of the physiologic side chain interactions and the conformation of the physiologic target, specificity is quite low [5-7]. And, the relatively limited specificity achievable with this approach is further compromised by the broad reactivity of the functional groups placed in the $\mathrm{P}_{1}{ }^{\prime}$ position: such activity-based probes can only reveal the presence of a reactive functional group or "active" site geometry, but not necessarily an enzymatic activity. Moreover, since protease probes of this design are not substrates, but suicide inhibitors, detailed measurements of enzyme kinetics are not possible. Additionally, these probes' limited cell permeability necessitates microinjection [8] or use at concentrations elevated considerably beyond physiologic ranges [4].

Despite these drawbacks, this class of probes has been widely used to assess intracellular protease activities and, recently, has been expanded to in vivo imaging as well as various protease screening/discovery efforts [9]. One such study at the single cell level with a fluoromethylketone probe resulted in a warning for interpretation of exclusive interaction with desired targets due to "certain puzzling observations" that "could not be easily explained by the initially proposed mechanism of binding" [10]. Although specificity has been improved [11], off-target enzyme staining persists as molecular complexity at the single cell

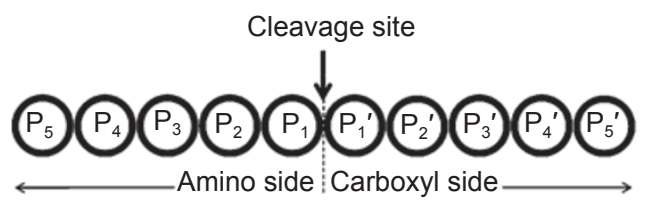

Figure 1 Nomenclature for substrates. Each circle represents the position of an amino acid in a target's recognition/cleavage site.

level both in vitro and in vivo presents staining targets not extant in solution, making chemical characterization of tagged targets essential.

Another approach to protease probe design does include sequence information from both sides of the cleavage site. Here, two fluorophores with complementary spectral properties are used such that the emission of one, nominally the donor, is quenched by the second, nominally the acceptor [12]. Upon cleavage, quenching of the fluorescence of the nominal donor is abolished such that monitoring of this fluorophore's emission intensity can serve as a measure of proteolytic activity $[13,14]$. In some probes the acceptor is replaced with a nonfluorescent chromophore [15]. (While the idea of heterolabeling a sequence from a known recognition/cleavage site remains a valid tool for protease activity quantitation, particularly in solution, a re-evaluation of the quenching mechanism has indicated a broader mechanism, not limited to heterolabeling [16]). Drawbacks of this particular approach include lack of cell 
membrane permeability of most known probes and where the acceptor is a fluorophore the necessity to add filters to most instrumentation.

In order to address the limited permeability problem, elements of each of the above two approaches have been combined with the HIV-1 TAT protein transduction domain (PTD) $[17,18]$, a decapeptide that has been useful for delivery of a wide variety of biologically active cargos. In one study a construct containing the TAT-PTD sequence, a tetrapeptide from the amino side of a target recognition/cleavage site, and both a donor and a quencher was synthesized as a probe for proteases inside live cells [19]. The TAT-PTD is believed to transport macromolecules into cells by an initial interaction with cell membrane lipid rafts in a receptor-independent manner, followed by a rapid internalization through macropinocytosis, then a $\mathrm{pH}$ drop and destabilization of the integrity of the macropinosome vesicle lipid bilayer, and, ultimately, release of the TATcargo into the cytosol [20]. Due to the strong electrostatic interaction between the basic (eight of the ten amino acids are arginine or lysine) TAT-PTD and the negatively charged cell-surface constituents plus the local cytosolic $\mathrm{pH}$ changes required for release, questions regarding cellular viability have arisen. Additionally, uniformity with respect to the percentage of cells taking up the probe and the subcellular distribution of the probe need to be evaluated. The latter is critical since many proteases in live cells are not located in the cytosol and, therefore, probes must be capable of crossing intracellular membranes.

\section{Design of cell permeable probes with enhanced speci- ficity and high cell permeability}

In order to address the above-described problems, amino acid sequences consisting of 4 to 12 residues spanning physiologic macromolecular recognition/cleavage sites have been synthesized and incorporated into peptides or peptidyl backbones [16, 21, 22]. Each substrate which contains 12 to 31 amino acids in toto is then homodoubly labeled near its ends with a fluorophore of significant transition dipole strength such that the two covalently-bound fluorophores form an intramolecular noncovalent H-type excitonic dimer [23-25] (Figure 2). Substrates which thereby assume loop-like structures allow for exceptionally high recognition by their cognate proteases due to the presence of not only linear sequences but also conformations that are found in native macromolecules. Notably, cell-permeable probes for members of the four (serine, cyteine, metallo-, and aspartyl) major protease classes have been synthesized and shown to have remarkably high specificity [26].

Solution studies

Using probes with the design depicted in Figure 2, pro- tease activities have been measured both in solution and in live cells and tissues. An example of a solution measurement is shown in Figure 3 where ElastoLux ${ }^{\circledR}$, a substrate containing the amino acid sequence Ala-Ile-Pro-Nle-Ser-Ile (cleavage between Nle and Ser), based on the cleavage site of nature's best elastase inhibitor, $\alpha_{1}$-antitrypsin, serves as a model substrate. Fluorescence intensity which increases as a function of time after addition of the serine protease elastase was validated by high performance liquid chromatography (HPLC) to correspond to the first cut in the substrate backbone, i.e., between the Nle and Ser residues [16]. Furthermore, spectroscopic data confirmed the presence of the intramolecular dimer in the intact substrate

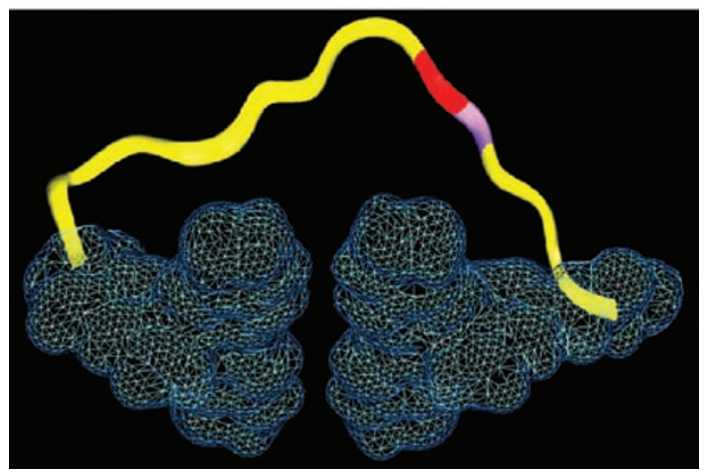

Figure 2 Model structure of cell permeable homodoubly-labeled fluorogenic protease substrates. The yellow ribbon represents the peptide or peptidyl backbone with the cleavage site between red and purple. Both ends are covalently labeled with the same fluorophore; the latter two form an intramolecular dimer. Presence of the dimer results in fluorescence quenching which is relieved upon cleavage of the backbone.

and its destruction upon cleavage of the peptide backbone between these two residues.

\section{Apoptosis: activation of caspase cascades}

One of the long-standing problems in protease substrate design has been how to design substrates which can discriminate among members of a closely evolved protease family. An example of the importance of the latter can be found in the voluminous literature of apoptosis which is briefly described as follows: all cells are believed to contain programs which when activated can lead to their own death. The major biochemical pathways of activation include a group of proteases, the caspases, which are characterized by both a catalytic cysteinyl residue and a strong preference for an aspartyl residue in the $\mathrm{P}_{1}$ position of their substrate recognition sequences. The order of activation of the $c a$. 


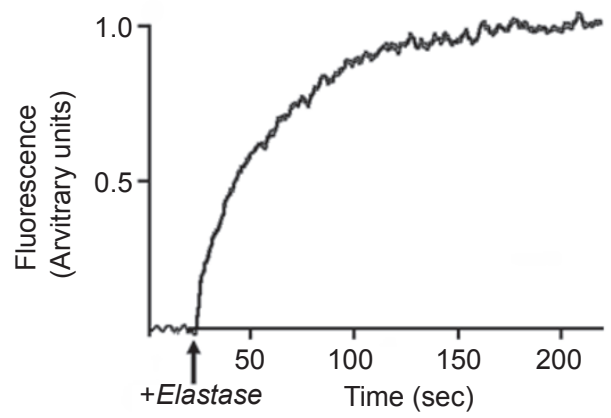

Figure 3 Solution study of substrate cleavage. The serine protease elastase cleaves the substrate ElastoLux ${ }^{\circledR}$ between norleucine and serine resulting in destruction of the intramolecular fluorophorefluorophore dimer and loss of quenching.

14 (identified) caspases, which depends on variables such as apoptogenic agent/event, cell type, and extracellular environment, has become an important area of study since the precise mechanism of programmed cell death will, one day, probably have profound consequences on treatment of many pathologic conditions. However, even with the extensive database of putative target sequences available [27], most of the caspase literature is based on substrates and inhibitors which do not clearly distinguish among the various caspase activities. This is largely due to one of the limitations of the substrate/inhibitor design described above: the use of tetrapeptides which contain only amino acid sequences derived from one side (the amino side) of the recognition/cleavage sites in known caspase targets or from combinatorial protease substrate sequence determination/optimization methods [28, 29]. Thus, the use of incomplete protease recognition sequences in these reporters and the resultant lack of native conformations have led to poor discrimination among caspase family members.

To illustrate that the activities of members of this close family of proteases can be clearly distinguished, five octadecapeptides were synthesized targeting five different caspases (caspases 1, 3, 6, 8, and 9) by incorporating sequences from both sides of the recognition/cleavage sites for each caspase [30]. In cases where the physiologic target was known, the full amino acid sequence through the cleavage site was incorporated; for caspase 9 where only amino terminal sequence information was available, the reported optimal tetrapeptide sequence, LEHD, was incorporated with a common carboxyl side sequence. Recombinant caspase 3 was then added to solutions containing each fluorogenic substrate at $1 \mu \mathrm{M}$. The data in Figure 4 clearly show that only the substrate containing the sequence DEVDGI, which was derived from poly(ADP-ribose) polymerase (PARP), a physiologic target for caspase 3, was cleaved between DEVD and GI.
Then, a direct comparison of the specificity between substrates for two distinct caspases, caspase 3 and caspase 6 , was made (Figure 5). Here, the two substrates were derived based on sequence information from both sides of the recognition/cleavage sites of two different physiologic targets, PARP for caspase 3 and nuclear lamin B for caspase 6. Recombinant caspases 3 and 6 were first titrated to yield the same cleavage rate using the caspase 3 tetrapeptide aminofluoromethylcoumarin substrate (DEVD-AFC) (20 $\mu \mathrm{M}$ ). With these titrated concentrations ( $1 \mathrm{nM}$ for caspase 3 and $5 \mathrm{nM}$ for caspase 6) substrate cleavage rates of the respective specific substrates were then measured. Even though the two caspases at their titrated concentrations yielded the same rate of cleavage of the linear tetrapeptide-fluorophore substrate, a clear discrimination between the two caspases was possible with homodoubly labeled substrates containing loop conformations.

\section{Live cell studies}

When an intact substrate with this design is added to a suspension or monolayer of cells in culture, the probe crosses all, i.e., the plasma as well as intracellular organelle, membranes by passive diffusion (Figure 6A). Once a substrate is recognized and cleaved by its cognate protease, the cleaved fragments are trapped on the side of the membrane where the protease activity resides $[30,31]$. (There is a low, but finite, diffusion rate of fragments across membranes.)

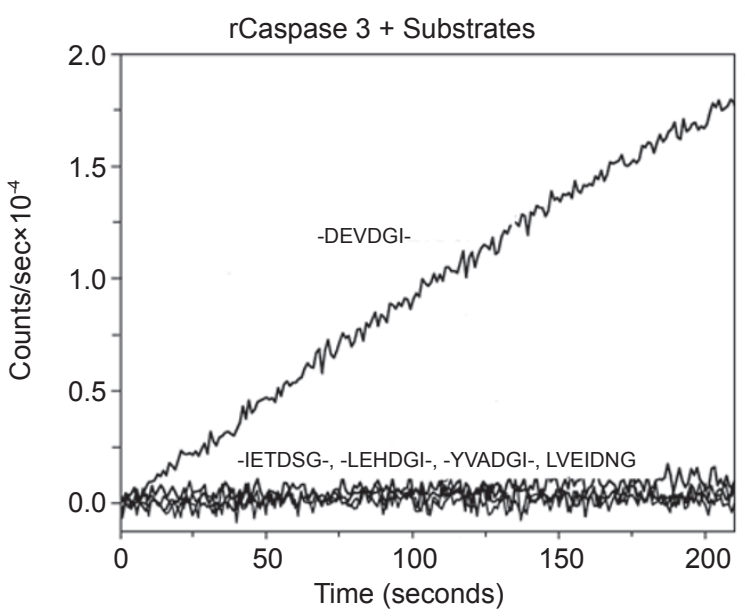

Figure 4 Recombinant caspase 3 cleaves only the substrate derived from its physiologic target, PARP. Of five different substrates derived from five different caspase target sequences, -DEVD'GI- (caspase 3), -LEHD'GI- (caspase 9), -IETD'SG(caspase 8), -YVAD'GI- (caspase 1), and -LVEID'NG- (caspase 6), only the caspase 3 target sequence was cleaved by recombinant caspase 3 . 
Enhanced Discrimination

between Caspases 3 and 6 Substrates

by $r$-Caspase 3 and $r$-Caspase 6

Titraed enzyme concentrations to yield same cleavage rate of DEVD-AFC substrate: rCaspase $6=5 \mathrm{nM}$

rCaspase $3=1 \mathrm{nM}$
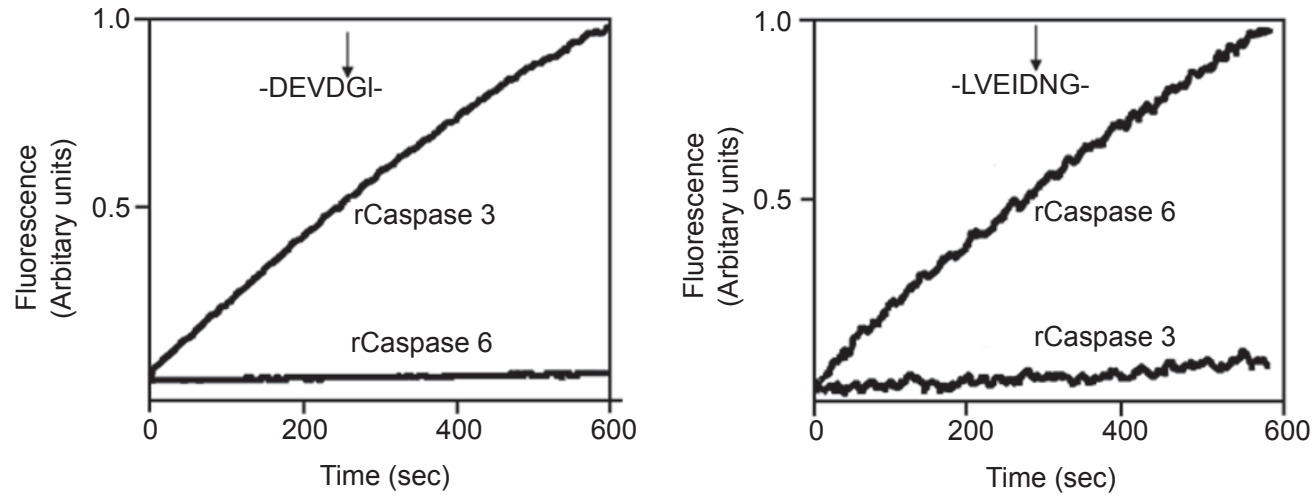

[Substrate $]=1 \mu \mathrm{M}$

Figure 5 Criss-cross specificity comparison between rCaspase 3 and rCaspase 6. rCaspase 6 cleaved the sequence (-LVEID'NG-) from the caspase 6 target nuclear lamin B but not from the Caspase 3 target (-DEVD'GI-) PARP while rCaspase 3 showed the opposite specificity.

It has been found that the intact probes can diffuse across membranes from a vast variety of cell types ranging from yeast to plant embryos and mammalian organisms. Figure 6 shows flow cytometry and confocal microscopy data from cells treated with apoptogenic agents that are known to activate caspase cascades by either intrinsic or extrinsic pathways. By flow cytometry (Figure 6A) the bimodality which is characteristic of cell populations being induced to undergo apoptotic death can be observed. Significantly, exclusion of the cell impermeant dye propidium iodide (PI) confirmed the integrity of all plasma membranes [32]. Confocal microscopy imaging (Figure 6B) illustrates the differential time of activation and subcellular localization of three different caspases (caspase 9, 6, and 3 in green, red, and blue, respectively). As activation of individual caspases is microenvironment-dependent, accurate subcellular localization is critically important. Thus, use of cell-permeable probes with the capability of discriminating among closely related proteases in live cell environments is essential for not only accurate mechanistic evaluation of the apoptotic process but also drug targeting. (In topics beyond the scope of this manuscript, this probe design is being utilized for protease inhibitor and drug delivery studies.)

\section{Cell-mediated cytotoxicity}

The ability of a cytotoxic lymphocyte, e.g., a $\mathrm{CD} 8^{+}$ cytotoxic lymphocyte (CTL) or natural killer (NK) cell, to destroy a target cell, e.g., a tumor or virally-infected cell, has been a subject of intense interest to immunologists for considerable time. For almost forty years, the gold standard for measuring this type of activity has been the ${ }^{51} \mathrm{Cr}$ release assay [33]. Unfortunately, the latter is deficient in many ways [34]: (1) Target cells must be loaded with a radioactive isotope, $\mathrm{Na}_{2}{ }^{51} \mathrm{CrO}_{4}$, with loading, per se, being inconsistent with respect to both individual cells and cell types. In fact, many, particularly primary, cells do not take up this label to any workable level. (2) The assay is quantitated by release of ${ }^{51} \mathrm{Cr}$ from targets following addition of effectors; the readout which is from the bulk cellular population not only results in high backgrounds but also precludes single cell quantitation. Additionally, multiplex readouts such as phenotyping individual dying target cells are not possible. However, the third and most critical shortcoming of the ${ }^{51} \mathrm{Cr}$ release assay as well as recent attempts to utilize more modern instrumentation, e.g., flow cytometry, is that all rely on physical destruction of the target cell plasma membrane. Importantly, destruction of membrane permeability is not a direct biochemical activity of cytotoxic lymphocytes. For example, the PI signal from intercalation into target cell DNA, which is used as a readout in many cytotoxicity studies, is a relatively late consequence of the plasma membrane permeability increase (vide supra) generated by the lethal hit from effectors. With this background the above-described probe design was applied toward quantitating a very early biochemical event in cell-mediated 
A
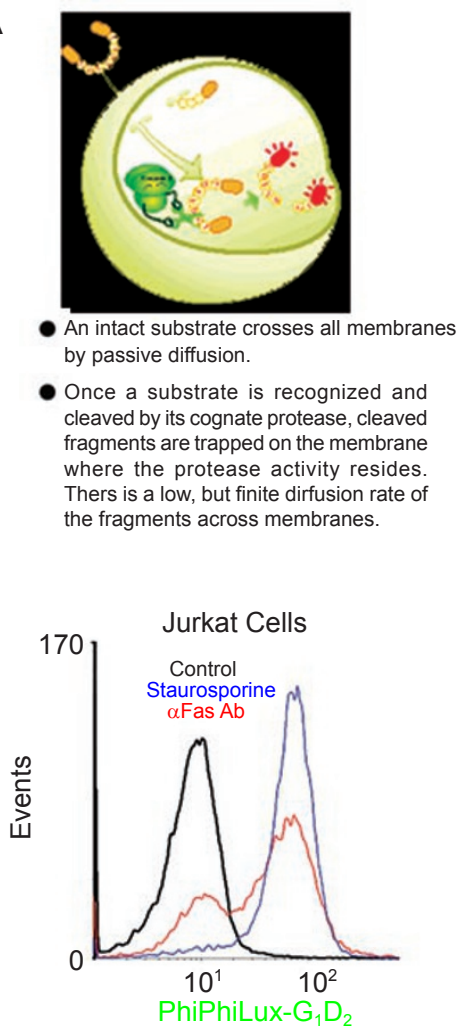

B

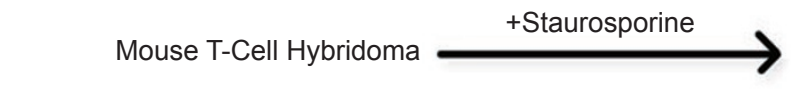
-LEHDGIN-LVEIDNG-DEVDGID-
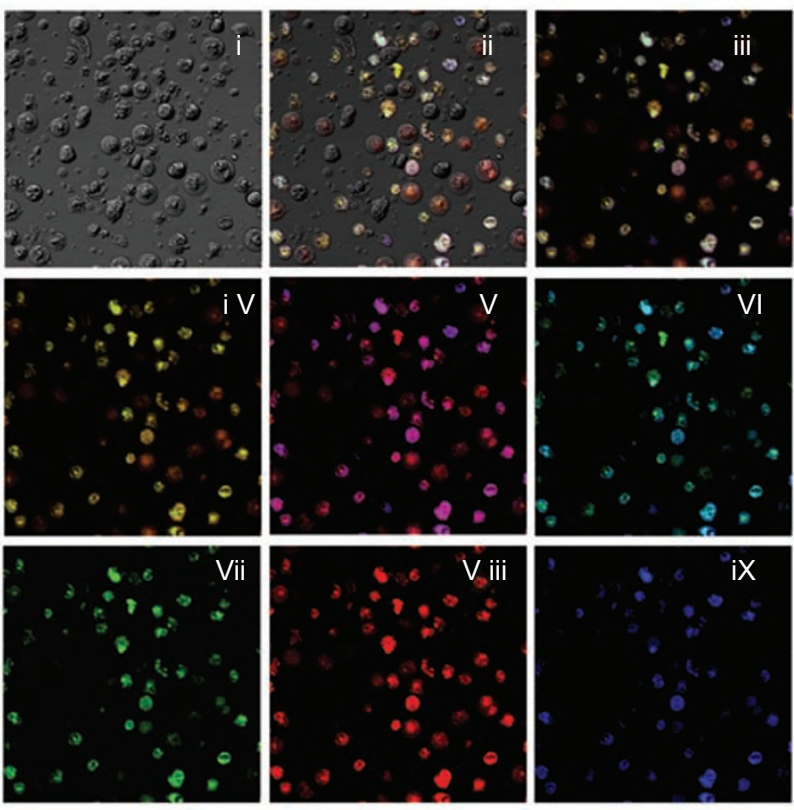

Figure 6 Cell permeable substrates enable live cell protease activity measurements by both microscopy and flow cytometry. (A) Induction of apoptosis in Jurkat cells by the intrinsic apoptogen staurosporine shows a ca. 1 log increase in fluorescence intensity per cell due to cleavage of the caspase 3 substrate PhiPhiLux ${ }^{\circledR}$ and a bimodal distribution following induction by the extrinsic apoptogen anti-Fas antibody. (B) Activation of caspases 9 (-LEHD'GI-), 6 (-LVEID'NG-), and 3 (-DEVD'GI-) in green, red, and blue, respectively, showing subcellullar localization unique for each activity. Images are: (i) DIC (differential interference contrast) (ii) DIC and caspases 9, 6, and 3 (iii) caspases 9, 6, and 3 (iv) caspases 9 and 6 (v) caspases 6 , and 3 (vi) caspases 9 and 3 (vii) caspase 9 (viii) caspase 6 and (ix) caspase 3.

cytotoxicity, namely, entry of the serine protease granzyme $\mathrm{B}$ (GzB) into target cells [35].

Although effector cells synthesize GzB, it is proteolytically inactive in CTLs and NK cells due to the acidic $\mathrm{pH}$ of their cytoplasmic granules where this protease, perforin and other components believed to be essential for effector cell assault on targets are stored. However, once GzB enters the cytosol of target cells where the $\mathrm{pH}$ is neutral, the molecule becomes enzymatically active and is able to process target cell procaspases. Therefore, a cell permeable fluorogenic probe derived from the physiological macromolecular substrate for GzB, DNA-dependent protein kinase catalytic subunit, was synthesized to serve as a direct biochemical measure of CTL/NK activity. The appearance of GzB activity in the cytoplasm of target cells can be used at the single cell level to both quantitate and image attack by effector cells.

In this assay (Figure 7), target cells are first labeled with a probe whose fluorescence is complementary to that of the cleaved GzB substrate. Effectors are then added in the presence of the substrate, cells are coincubated for a defined time, typically, one hour, and then examined at the single cell level by flow cytometry, microscopy, or other cytometric instrumentation (Figure 7A). Measurement of the lethal hit on target cells by effectors prior to changes in target membrane permeability (Figure 7B) which both ${ }^{51} \mathrm{Cr}$ release and the earlier flow cytometry methodologies depend upon is enabled. Figure 8 shows quantitation of GzB activity delivered from either CTLs or NK cells into the cytoplasm of three different targets (Jurkat, K562, and Daudi cells). The assay has also been used with primary cells to assess suppressed cytolytic activity from subsets of lymphocytes in immunocompromised (AIDS) patients [36], to identify human tumor targets for NK cells [37], and to study T-cell regulation of cytotoxicity [38].

In addition to measuring the introduction of a molecule capable of inducing a lethal hit inside a live target cell, the caspase substrates originally designed for single cell quantitation following apoptogenic induction by classical intrinsic or extrinsic apoptogens can be utilized for confirmation of 
the lethality of a GzB hit [39-42]. Furthermore, simultaneous use of complementarily labeled cell permeable fluorogenic substrates for $\mathrm{GzB}$ and caspase 3 has indicated that GzB activity precedes caspase 3 activity inside every cell examined by both flow cytometry and microscopy [35]. Also, as previous studies had provided conflicting data for the effect of the anti-apoptotic factor Bcl-2 in target cells, the apoptotic pathway utilized by $\mathrm{GzB}$ was examined. It was clearly shown by both activation of caspases following the introduction of GzB and the appearance of the morphologic hallmarks of apoptosis, i.e., membrane blebbing and transient swelling, that Bcl-2 is unable to limit cell death mediated by cytotoxic lymphocytes. These results point to the death pathway initiated by entry of GzB into target cells short-circuiting the well-documented intrinsic and extrinsic caspase cascade activation and amplification pathways.

\section{Antibody-Dependent Cellular Cytotoxicity (ADCC)}

The biotechnology revolution has given rise to the emergence of monoclonal antibody (MAb) therapeutics. Although these macromolecules have found utility in the management of several malignant disease conditions, their mechanism(s) of action in most remains unclear. It is believed that at least three modes of target cell destruction are possible: complement-mediated pathway, macrophage phagocytosis of opsonized tumor cells, and cell-mediated cellular cytotoxicity (ADCC) [43]. While the former two have been studied with many classical assays and, certainly, complement and opsonization and their sequelae are "players" in MAb therapeutics, cellmediated cytotoxicity dependent on an antibody has not been well-characterized at the molecular level. Thus, it is now quite timely that, with the availability of a live cell GzB activity assay, MAbs be directly analyzed for their ability to effect the interaction between NK and tumor cells in screening (Figure 9) as well as in longitudinal studies throughout the course of an individual patient's therapy. The assay is particularly useful for the latter in determining variations in therapeutic efficacy of a monoclonal antibody such as Rituxan as characteristics of a cancer under treatment can change [44, 45]. Furthermore, insight into the true effector mechanisms of monoclonal antibodies used for inflammatory conditions such as rheumatoid arthritis [46] and vasculitis [47] as well as in anti-T cell antibody therapy used to prevent graft versus host disease (GVHD) subsequent to bone marrow transplantation [48, 49] may also be achieved with studies of the molecular sequelae to the initial binding of antibodies.

\section{Conclusions}

In studies described above, it has been shown that by combining probes with complementary spectral properties and discriminatory capability for a variety of protease activities, insight into interacting and dependent pathways can be gained. The significance of probing biochemical activities in environments where the molecular composition and organization accurately represent physiologic states impacts the type and depth of conclusions that can be drawn from the acquired data. Since patterns of activation of protease cascades are believed to be emblematic of pathophysiologic states including cancer metastasis, neurodegeneration, and
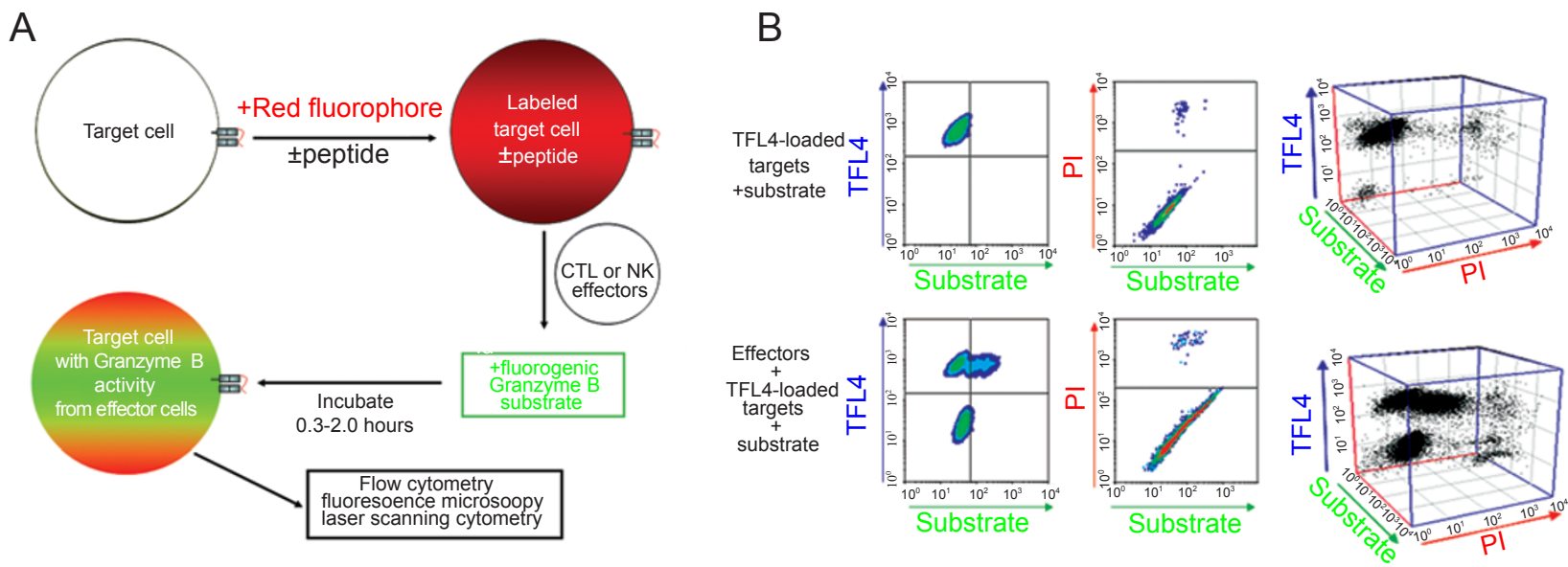

Figure 7 Cell-mediated cytotoxicity measured at the single cell level using a cell-permeable GzB substrate. (A) Measurement of GzB activity exclusively in target cells is carried out after coincubation of prelabeled targets with effectors (NK or CTL) in the presence of a cell permeable GzB substrate. (B) GzB ${ }^{+}$target cells have intact permeability as indicated by exclusion of propidium iodide $(\mathrm{PI})$ (in contrast to ${ }^{51} \mathrm{Cr}$ release and other flow cytometry-based cytotoxicity assays). 
$\%$ Granzyme B+ targets
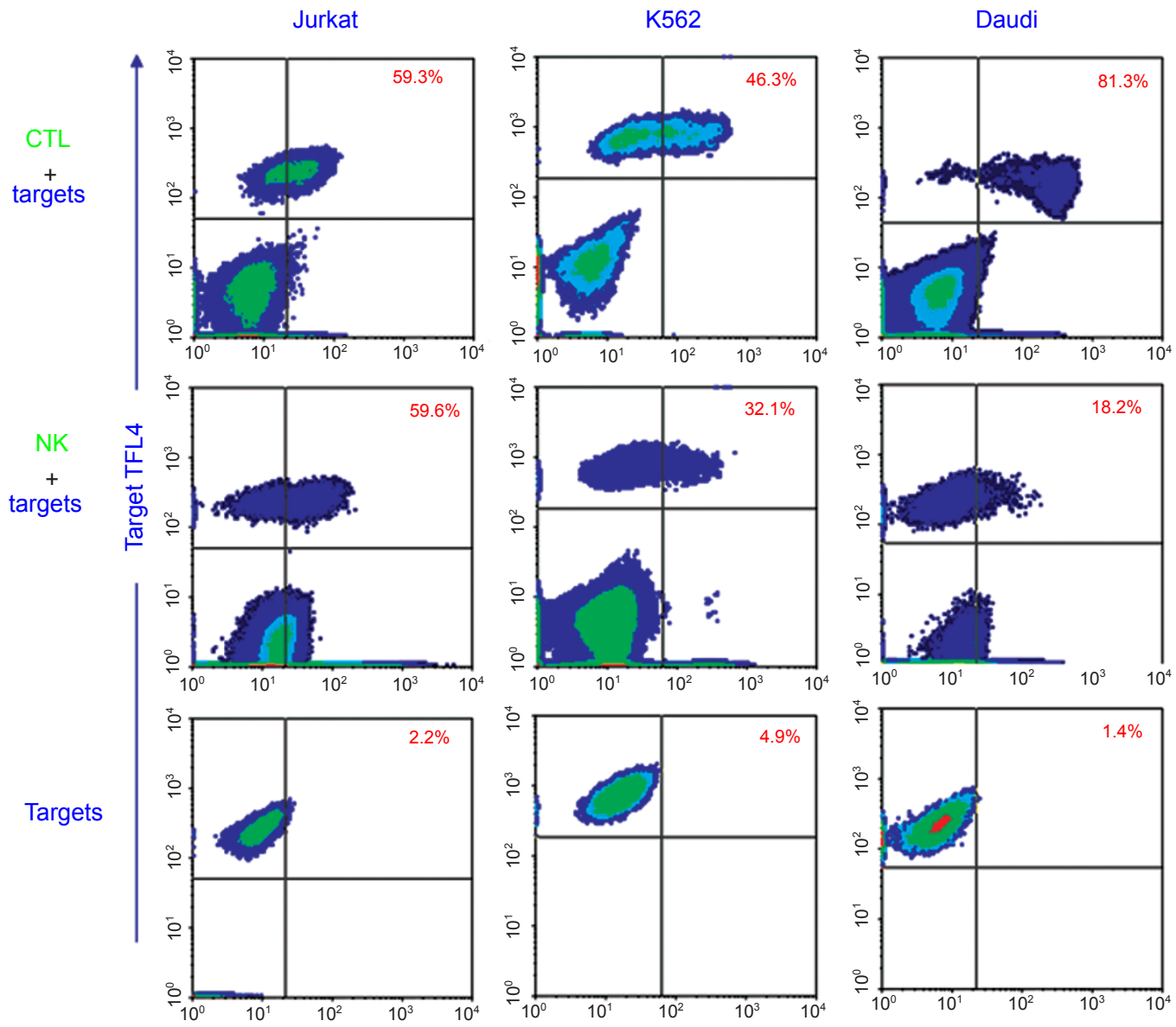

Granzyme B Activity

Figure 8 Live single cell-mediated cytotoxicity assays with multiple targets and effectors. Jurkat (right column), K562 (middle column), and Daudi (right column) cell lines served as targets for both CTL (top row) and NK (middle row) cells. Targets alone are shown in the bottom row.

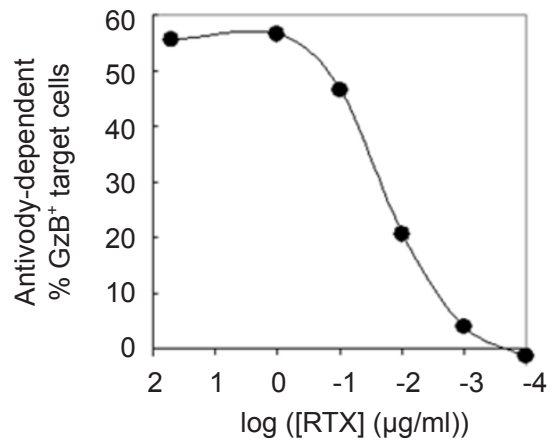

Figure 9 Live single cell measurement of antibody-dependent cellular cytotoxicity (ADCC). The increased presence of GzB activity inside target (Raji) cells after coincubation with $\mathrm{CD} 16^{+} \mathrm{NK}$ cells is shown to be a function of the concentation of the anti-CD20 monoclonal antibody Rituxan (Compliments of E Kuta). Conditions were: Effector:Target $=2: 1,1 \mathrm{~h}$ coincubation . inflammation, measurements with probes having high specificity and access to physiologically representative environments are essential.

\section{References}

1 Nair R, Rost B. Better prediction of sub-cellular localization by combining evolutionary and structural information. Proteins 2003; 53:917-30.

2 Mann M, Jensen ON. Proteomic analysis of post-translational modifications. Nat Biotechnol 2003; 21:255-61.

3 Richardson PL. The determination and use of optimized protease substrates in drug discovery and development. Curr Pharm Design 2002; 8:2559-2581.

4 Van Noorden CJF. The history of Z-VAD-FMK, a tool for understanding the significance of caspase inhibition. Acta Histochemica 2001; 103:3241-3251. 
5 Schotte PW, Declercq S, Van Huffel P, et al. Non-specific effects of methyl ketone peptide inhibitors of caspases. FEBS Lett 1999; 442:117-121.

6 Chauvier D, Ankri S, Charriaut-Marlangue C, et al Broad-spectrum caspase inhibitors: from myth to reality? Cell Death Differ 2006; 14:387-391.

7 Berger AB, Sexton KMB, Bogyo M. Commonly used caspase inhibitors designed based on substrate specificity profiles lack selectivity. Cell Res 2006; 16:961-963.

8 Mizukami S, Kikuchi K, Higuchi T, et al. Imaging of caspase-3 activation in HeLa cells stimulated with etoposide using a novel fluorescent probe. FEBS Lett 1999; 453:356-360.

9 Fonovic M, Bogyo M. Activity based probed for proteases: applications to biomarker discovery, molecular imaging and drug screening. Curr. Pharm. Design 2007; 13:253-261.

10 Pozarowski P, Huang X, Halicka DH, et al. Interactions of fluorochrome-labeled caspase inhibitors with apoptotic cells: A caution in data interpretation. Cytometry A. 2003; 55:50-60.

11 Berger AB, Witte MD, Denault J-B, et al. Identification of early intermediates of caspase activation using selective inhibitors and activity-based probes. Mol Cell 2006; 23:509-521.

12 Forster T. Intermolecular energy migration and fluorescence. Ann Phys 1948; 2:55-75.

13 Latt SA, Auld DS, Vallee BL. Fluorescence determination of carboxypeptidase A activity based on electronic energy transfer. Anal Biochem 1972; 50:56-62.

14 Carmel A, Zur M, Yaron A, et al. Use of substrates with fluorescent donor and acceptor chromophores for the kinetic assay of hydrolases. FEBS Lett 1973; 30:11-13.

15 Matayoshi ED, Wang GT, Krafft GA, et al. Novel fluorogenic substrates for assaying retroviral proteases by resonance energy transfer. Science 1990; 247:954-958.

16 Packard BZ, Toptygin DD, Komoriya A, et al. Profluorescent protease substrates: intramolecular dimers described by the exciton model. Proc Natl Acad Sci USA 1996; 93:11640-11645.

17 Frankel A, Pabo C. Cellular uptake of the Tat protein from human immunodeficiency virus. Cell 1988; 55:1189-1193.

18 Green M, Loewenstein P. Autonomous functional domains of chemically synthesized human immunodeficiency virus Tat trans-activator protein. Cell 1988; 55:1179-1188.

19 Bullok K, Piwnica-Worms D. Synthesis and characterization of a small, membrane-permeant, caspase-activatable far-red fluorescent peptide for imaging apoptosis. J Med Chem 2005; 48:5404-5407.

20 Wadia JS, Stan RV, Dowdy SF. Transducible TAT-HA fusogenic peptide enhances escape of TAT-fusion proteins after lipid raft macropinocytosis. Nature Med 2004; 10:310-315.

21 Packard BZ, Toptygin DD, Komoriya A, et al. The design of fluorogenic protease substrates guided by exciton theory. Meth Enzym 1997; 278:15-28.

22 Packard BZ, Komoriya A, Nanda V, et al. Intramolecular excitonic dimers in protease substrates: modification of the backbone moiety to probe the H-dimer structure. J Phys Chem B 1998; 102:1820-1827.

23 Packard BZ, Komoriya A, Toptygin DD, et al. Structural characteristics of fluorophores which form intramolecular H-type dimers in a protease substrate. $J$ Phys Chem B 1997; 101:5070-5074.

24 Packard BZ, Toptygin DD, Komoriya A, et al. Characterization of fluorescence quenching in bifluorophoric protease substrates.
Biophys Chem 1997; 67:167-176.

25 Packard BZ, Toptygin DD, Komoriya A, et al. Intramolecular resonance dipole-dipole interactions in a protease substrate. $J$ Phys Chem B 1998; 102:752-758.

26 Packard BZ, Komoriya A. A Method in Enzymology for Measuring Intracellular Protease Activities in Live Cells. Meth Enzym (in press)

27 Nicholson DW, Thornberry NA. Caspases: killer proteases. Trends Biochem Sci 1997; 22:299-306.

28 Harris JL, Peterson EP, Hudig D, et al. Definition and redesign of the extended substrate specificity of granzyme B. J Biol Chem 1998: 273:27364-27373.

29 Thornberry NA, Rano TA, Peterson EP, et al. A Combinatorial Approach Defines Specificities of Members of the Caspase Family and Granzyme B. J Biol Chem 1997; 272:17907-17911.

30 Komoriya A, Packard BZ, Brown MJ, et al. Assessment of caspase activities in intact apoptotic thymocytes using cellpermeable fluorogenic caspase substrates. J Exp Med 2000; 191:1819-1828.

31 Packard BZ, Komoriya A, Brotz TM et al. Caspase 8 activity in membrane blebs after anti-Fas ligation. J Immunol 2001; 167:5061-5066.

32 Telford WG, Komoriya A, Packard BZ. Multiparametric analysis of apoptosis by flow and image cytometry. Methods Mol Biol 2004; 263:141-160.

33 Brunner KT, Mauel J, Cerottini JC, et al. Quantitative assay of the lytic action of immune lymphoid cells on ${ }^{51} \mathrm{Cr}$-labelled allogeneic target cells in vitro; inhibition by isoantibody and by drugs. Immunology 1968; 14:181-196.

34 Liu L, Chahroudi A, Silvestri G, et al. Visualization and quantification of $\mathrm{T}$ cell-mediated cytotoxicity using cell-permeable fluorogenic caspase substrates. Nat Med 2002; 8:185-189.

35 Packard BZ, Telford WG, Komoriya A, et al. Granzyme B activity in target cells detects attack by cytotoxic lymphocytes. $J$ Immunol 2007; 179:3812-3820.

36 Kinter A, McNally J. Riggin L, et al. Suppression of HIV-specific $\mathrm{T}$ cell activity by lymph node $\mathrm{CD} 25+$ regulatory $\mathrm{T}$ cells from HIV-infected individuals. Proc Natl Acad Sci USA 2007; 104:3390-3395.

37 Carlsten M, Bjorkstrom NK. Norell H et al. DNAX accessory molecule-1 mediated recognition of freshly isolated ovarian carcinoma by resting natural killer cells. Cancer Res 2007; 67:1317-1325.

38 Tarasenko T, Kole HK, Chi AW, et al. T cell-specific deletion of the inositol phosphatase SHIP reveals its role in regulating Th1/Th2 and cytotoxic responses. Proc Natl Acad Sci USA 2007; 104:11382-11387.

39 Liu L, Packard BZ, Brown MJ, et al. Assessment of lymphocytemediated cytotoxicity using flow cytometry. Methods Mol Biol 2004; 263:125-140.

40 Barber DL, Wherry EJ, Ahmed R. Cutting Edge: Rapid In Vivo Killing by Memory CD8 T Cells. J Immunol 2003; 171:2731.

41 Harcourt J, Alvarez R, Jones LP, et al. Respiratory syncytial virus $\mathrm{G}$ protein and $\mathrm{G}$ protein $\mathrm{CX} 3 \mathrm{C}$ motif adversely affect $\mathrm{CX} 3 \mathrm{CR} 1+$ T cell responses. J Immunol 2006; 176:1600-1608.

42 Chen J,. Ellison FM, Eckhaus MA, et al. Minor antigen H60mediated aplastic anemia is ameliorated by immunosuppression and the infusion of regulatory T cells. J Immunol 2007; 178:4159- 
4168.

43 Taylor RP, Lindorfer MA. Drug Insight: the mechanism of action of rituximab in autoimmune disease - the immune complex decoy hypothesis. Nat Clin Pract Rheumatol 2007; 3:86-95.

44 Coiffier B. Monoclonal antibody as therapy for malignant lymphomas. C R Biologies 2006; 329:241-254.

45 Collins-Burow B, Santos ES. Rituximab and its role as maintenance therapy in non-Hodgkin lymphoma. Expert Rev Anticancer Ther 2007; 7:257-273.

46 Bayry J, Sebastien L-D, Kazatchkine MD, et al. Monoclonal antibody and intravenous immunoglobulin therapy for rheumatic diseases: rationale and mechanisms of action. Nat Clin Pract Rheumatol 2007; 3:262-272.

47 Hellmann DB, Imboden JB. Update in rheumatology. Ann Intern Med 2006; 145:834-838.

48 Mathew J, DeFaria W, Kato T, et al. Abrogation of the Alloreactive Responses of Cadaveric Donor Intestinal Lymphocytes by Intraoperative Campath-1H Exposure. Transplant Proc 2005; 37:1375-8.

49 Marks R, Finke J. Biologics in the prevention and treatment of graft rejection. Springer Semin. Immunopathol 2006; 27:457476. 